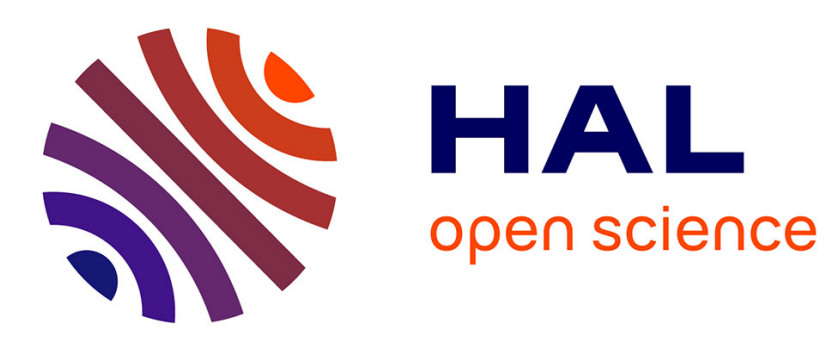

\title{
Attosecond correlation dynamics during electron tunnelling from molecules
}

\author{
Zachary B Walters, Olga Smirnova
}

\section{To cite this version:}

Zachary B Walters, Olga Smirnova. Attosecond correlation dynamics during electron tunnelling from molecules. Journal of Physics B: Atomic, Molecular and Optical Physics, 2010, 43 (16), pp.161002. 10.1088/0953-4075/43/16/161002 . hal-00597865

\section{HAL Id: hal-00597865 \\ https://hal.science/hal-00597865}

Submitted on 2 Jun 2011

HAL is a multi-disciplinary open access archive for the deposit and dissemination of scientific research documents, whether they are published or not. The documents may come from teaching and research institutions in France or abroad, or from public or private research centers.
L'archive ouverte pluridisciplinaire HAL, est destinée au dépôt et à la diffusion de documents scientifiques de niveau recherche, publiés ou non, émanant des établissements d'enseignement et de recherche français ou étrangers, des laboratoires publics ou privés. 


\title{
Attosecond correlation dynamics during electron tunneling from molecules
}

\author{
Zachary B. Walters
}

Max Born Institute, Max Born Strasse 2a, 12489 Berlin Adlershof, Berlin Germany

E-mail: zwalters@gmail.com

\section{Olga Smirnova}

Max Born Institute, Max Born Strasse 2a, 12489 Berlin Adlershof, Berlin Germany

E-mail: Olga.Smirnova@mbi-berlin.de

\begin{abstract}
We present analytical theory of strong field ionization of molecules, which takes into account rearrangement of multiple interacting electrons during the ionization process. We show that such rearrangement offers an alternative pathway to the ionization of orbitals more deeply bound than the Highest Occupied Molecular Orbital (HOMO). This pathway is not subject to the full exponential suppression characteristic of the direct tunnel ionization from the deeper orbitals. The departing electron produces an "attosecond correlation pulse" which controls the rearrangement during the tunneling process. The shape and duration of this pulse are determined by the electronic structure of the relevant states, molecular orientation, and the laser parameters.
\end{abstract}

PACS numbers: $42.50 . \mathrm{Hz}, 32.80 . \mathrm{Rm}, 33.80 . \mathrm{Wz}$

Keywords: multichannel strong field ionization, attosecond multielectron dynamics, core rearrangement, molecular orbitals 
Tunnel ionization in static electric fields is exponentially sensitive to ionization potential $I_{p}$ : ionization from deeper bound states is exponentially suppressed. Similar suppression is commonly expected during ionization in intense low-frequency laser fields [1]. It has thus been a natural and nearly universal assumption that only the least bound electron may escape the atom or molecule, leaving the resulting ion in its lowest electronic state. We show that the conventionally assumed exponential suppression of ionization rates is strictly valid only if the state of the ion is frozen during the tunneling process. The ionization dynamics change if the state of the ion is allowed to evolve, reflecting the interaction between the departing electron and the ionic core. We show that correlated electron dynamics during ionization provides a new pathway to the creation of excited states of the ion, which is not subject to the full exponential suppression accompanying direct tunneling from a more deeply bound state.

One would think that the importance of excited electronic states of the ion during ionization could be easily verified by examination of photoelectron spectra. Indeed, different ionization channels, corresponding to different $I_{p}$ 's should lead to photoelectron peaks with different energy. While this is indeed the case for one-photon ionization, highly non-linear nature of strong-field processes make observation of the alternative ionization channels quite challenging in the strong field regime [2].

The first experiment [3] aimed at observing ionization channels with higher $I_{p}$ during strong-field ionization of molecules has been performed with $\mathrm{H}_{2}$. However, the large (about $20 \mathrm{eV}$ ) energy needed to excite the $\mathrm{H}_{2}^{+}$ion resulted in a vanishingly small $\left(10^{-6}\right)$ excited state population [3]. An important problem arising in such experiments is the need to separate the creation of excited ions during and after ionization. Recent studies of strong field ionization of hydrocarbon molecules [4] solved this problem by using covariance technique, correlating energies in the photoelectron spectra with the ionic fragments. These experiments have unambiguously demonstrated the contribution of several ionization channels corresponding to the population of different electronically excited states during ionization. Together with an avalanche of alternative experimental evidence $[5,6,7,8,9,10,11]$, the body of new experimental data poses challenges to the theory of strong field ionization.

While strong field ionization of atoms is well understood (see e.g. [13, 12]) in the single channel limit, a quantitative explanation of single channel ionization of molecules or an accurate treatment of multiple coupled channels represent problems at the frontier of strong field theory (see e.g. [14, 15, 16, 17, 18, 19, 20, 21, 22, 23]). The importance of channel coupling due to the laser field has been emphasized theoretically in $[9,20,23]$. Ref. [9] suggested the importance of electron correlations during tunnelling and provided the first evidence of non-trivial dynamics induced by this coupling.

We present a multichannel generalization of the strong field ionization theory originally proposed in the classic papers of Popov, Perelomov and Terent'ev (PPT)[13]. We include channel coupling induced by electronic interactions between the departing electron and the electrons left behind in the molecule. Interactions with the departing electron produce an "attosecond correlation pulse" which may drive transitions between 
ionic states during the tunnelling process. The shape and duration of this pulse are determined by (i) the electronic structure of the relevant states and (ii) the parameters of the laser field initiating ionization. The resulting transitions present alternative pathways to removing an electron from more deeply bound orbitals, which are not subject to the full exponential suppression of the direct pathway.

The full Hamiltonian of an $\mathrm{N}$ electron molecule interacting with a laser field is given by $H^{N}=T_{e}^{N}+V_{C}^{N}+V_{e e}^{N}+V_{l}^{N}, V_{C}^{N}=\sum_{m, i=1}^{i=N}\left|\mathbf{R}_{m}-\mathbf{r}_{i}\right|^{-1}, V_{e e}^{N}=\sum_{i \neq j}^{N}\left|\mathbf{r}_{i}-\mathbf{r}_{j}\right|^{-1}$, $V_{l}^{N}=\sum_{i} E(t) \cdot \mathbf{r}_{i}$, where the atomic nuclei are frozen at positions $\mathbf{R}_{m}$, index $m$ runs over the nuclei, superscript $N$ indicates the number of electrons involved, $T_{e}^{N}$ is the electron kinetic energy operator, $V_{C}^{N}$ describes the Coulomb potential of the nuclei, $V_{e e}^{N}$ describes the electron correlations, $V_{l}^{N}$ describes interaction with the laser field. It is convenient to define the Hamiltonian of the ion in the laser field $H^{N-1}=T_{e}^{N-1}+V_{C}^{N-1}+V_{e e}^{N-1}+V_{l}^{N-1}$, and the Hamiltonian for a single electron interacting with the laser field and the molecular ion $H^{e}=H^{N}-H^{N-1}$. The exact solution for the $\mathrm{N}$ electron wavefunction of a molecule, which starts from the ground electronic state $\Psi_{g}^{N}(\mathbf{r})$, can be written as [24]:

$$
\begin{aligned}
& \Psi^{N}(\mathbf{r}, t)=-\int_{0}^{t} \int_{0}^{t} d t^{\prime} d t^{\prime \prime} U^{N}\left(t, t^{\prime \prime}\right) V_{e e}\left(t^{\prime \prime}\right) U_{1}^{N}\left(t^{\prime \prime}, t^{\prime}\right) V_{l}^{N}\left(t^{\prime}\right) U_{0}^{N}\left(t^{\prime}, 0\right) \Psi_{g}^{N}(\mathbf{r})- \\
& -i \int_{0}^{t} d t^{\prime} U_{1}^{N}\left(t, t^{\prime}\right) V_{l}^{N}\left(t^{\prime}\right) U_{0}^{N}\left(t^{\prime}, 0\right) \Psi_{g}^{N}(\mathbf{r})+U_{0}^{N}(t, 0) \Psi_{g}^{N}(\mathbf{r}) .
\end{aligned}
$$

Here $V_{e e}=V_{e e}^{N}-V_{e e}^{N-1}$ describes the interaction of the departing electron with all the electrons in the ion. N-electron propagators $U_{0}^{N}$ and $U_{1}^{N}$ are determined as $i \dot{U}_{i}^{N}=H_{i}^{N} U_{i}^{N},(i=0,1)$, where $H_{0}^{N}$ is the field-free Hamiltonian of the molecule $H_{0}^{N}=H^{N}-V_{l}^{N}$ and $H_{1}^{N}=H^{N}-V_{e e}$.

Since ionization occurs in the presence of a strong laser field, it is necessary to introduce basis states which incorporate the effects of the laser field. Therefore, we write propagators in equation (1) using the basis of field-dressed (Floquet) electronic states of the ion $\left|\mathfrak{n}_{t}^{N-1}\right\rangle$ and channel specific one-electron continuum states $\left|\mathbf{k}_{t}^{n}\right\rangle$. The evolution of the $(N-1)$-electron Floquet states is governed by the Hamiltonian $H^{(N-1)}$. The Hamiltonian for the single electron includes a channel specific Hartree potential $V_{H}^{\mathfrak{n}}=\left\langle\mathfrak{n}_{t}^{N-1}\left|V_{e e}^{N}\right| \mathfrak{n}_{t}^{N-1}\right\rangle$, the Coulomb potential of the nuclei, and the laser field. Note that the Hartree potential is defined for field-dressed states of the ion and includes polarization of the core by the laser field.

Ionization amplitudes are found by projecting the $N$ electron wavefunction (1) onto a basis of $(N-1)$-electron ionic states and single electron continuum states. The integrals are calculated using the saddle point method, generalizing the approach of Ref.[13] to the multi-channel case. Just as in Ref.[13], the effect of the ionic core is included in the eikonal approximation, and the saddle points of these integrals are found in the zeroth order of the iterative saddle point method [28, 29]. The saddle point equations define a trajectory followed by the departing electron: $\left(\mathbf{k}_{f}+A\left(t_{0}\right)\right)^{2} / 2=-I_{p}$, $\mathbf{r}_{0}(t)=\int_{t_{0}}^{t}\left(\mathbf{k}_{f}+\mathrm{A}(\tau)\right) \mathrm{d} \tau$, where the laser electric field $\mathcal{E}(t) \equiv-\partial \mathrm{A}(\mathrm{t}) / \partial \mathrm{t}$. These conditions describe an electron which begins tunnelling at (complex) time $t_{0}$ and exits 
the subbarrier region at a time of birth on the real axis. $\dagger$

The final result for the ionization amplitude is the sum of direct and indirect terms:

$$
\left\langle\mathbf{k}_{f}^{m} \mathfrak{m}_{t}^{(N-1)} \mid \Psi^{N}(\mathbf{r}, t)\right\rangle=a_{\mathfrak{m} \mathfrak{m}}^{(d)}\left(\mathbf{k}_{f}^{m}\right)+\sum_{n} a_{\mathfrak{m} \mathfrak{n}}^{(i)}\left(\mathbf{k}_{f}^{m}\right)
$$

The direct and indirect ionization amplitudes are:

$$
\begin{array}{ll}
a_{\mathfrak{m}}^{(d)}\left(\mathbf{k}_{f}, t\right)=b_{\mathfrak{m m}}\left(t, t_{0}\right) a_{\mathfrak{m}}^{(P P T)}\left(\mathbf{k}_{f}, t\right), & b_{\mathfrak{m m}}=\left\langle\mathfrak{m}_{t}^{(N-1)}\left|U^{N-1}\left(t, t_{0}\right)\right| \mathfrak{m}_{t_{0}}^{(N-1)}\right\rangle, \\
a_{\mathfrak{m} \mathfrak{n}}^{(i)}\left(\mathbf{k}_{f}, t\right)=c_{\mathfrak{m} \mathfrak{n}}\left(\mathbf{k}_{f}, t, t_{0}\right) a_{\mathfrak{n}}^{(P P T)}\left(\mathbf{k}_{f}, t\right), & c_{\mathfrak{m} \mathfrak{n}}=-i \int_{t_{0}}^{t} d t^{\prime \prime}\left\langle\mathfrak{m}_{t^{\prime \prime}}^{N-1}\left|V^{e e}\left(\mathbf{r}_{0}\left(t^{\prime \prime}\right)\right)\right| \mathfrak{n}_{t^{\prime \prime}}^{N-1}\right\rangle
\end{array}
$$

Here $V^{e e}\left(\mathbf{r}_{0}\left(t^{\prime \prime}\right)\right)$ denotes electron-electron correlation operator $V^{e e}$ with the coordinate of the tunnelling electron substituted by the saddle-point subbarrier trajectory. The matrix elements from this operator calculated between the laser-dressed (Floquet) states of the ion $\left|\mathfrak{n}_{t}^{N-1}\right\rangle,\left|\mathfrak{m}_{t}^{N-1}\right\rangle$, determine the correlation potential:

$$
V_{\mathfrak{m} \mathfrak{n}}^{c o r r}(t)=\left\langle\mathfrak{m}_{t}^{N-1}\left|V^{e e}\left(\mathbf{r}_{0}(t)\right)\right| \mathfrak{n}_{t}^{N-1}\right\rangle,
$$

i.e. the effective field that induces transitions in the ion [30, 31]. Both direct and indirect ionization amplitudes are proportional to the standard PPT single-channel amplitude $a_{\mathfrak{l}}^{(P P T)}\left(\mathbf{k}_{f}, t\right)$, corresponding to the parent channel $\left|\mathfrak{l}_{t}\right\rangle$.

The direct amplitude $a_{\mathfrak{m}}^{(d)}\left(\mathbf{k}_{f}, t\right)$ corresponds to the traditional single channel ionization amplitude, in which an electron ionizes and propagates in the channel specific Hartree potential without causing the ion to change its state. The factor $b_{\mathfrak{m m}}\left(t, t_{0}\right)$ reflects a correction to the conventional tunneling amplitude due to laser-induced channel interaction. In the quasistatic limit, this corresponds to the Stark shift. In our analysis, the time integration is performed using complex time, and $b_{\mathfrak{m m}}\left(t, t_{0}\right)$ may be interpreted as the norm of Floquet states following non-Hermitian dynamics in the complex interval between $t$ and $t_{0}$ [27].

Our focus is the indirect amplitude $a_{\mathfrak{m} \mathfrak{n}}^{(i)}\left(\mathbf{k}_{f}\right)$ equation (4), which reflects changes in the ionic state due to electronic correlation during ionization. At time $t^{\prime \prime}$, interaction with the tunneling electron causes the ion to change its state from $|\mathfrak{n}\rangle$ to $|\mathfrak{m}\rangle$. The expression for the off-diagonal matrix element $c_{\mathfrak{m} n}$ shows that after $t^{\prime \prime}$, the tunneling electron propagates in the Hartree potential of the new state. Naturally, all $t^{\prime \prime}$ between $t_{0}$ and $t$ contribute to $c_{\mathfrak{m} \mathfrak{n}}$. The channel coupling is described in the lowest order with respect to electron correlations and in all orders with respect to the laser field.

Because $t_{0}$ satisfying the saddle point conditions will in general be complex, propagation of a state with positive $I_{p}$ to the real axis yields the familiar exponential suppression of tunneling with respect to energy. However, the expression for $c_{\mathfrak{m n}}$ involves not one but two propagation steps, from $t_{0}$ to $t^{\prime \prime}$ and from $t^{\prime \prime}$ to the real axis. Since the ion may change its state at time $t^{\prime \prime}$ due to the correlation potential, the indirect channel will have an exponential suppression which is intermediate between those of the more- and less deeply bound states. Excitations occurring at $t^{\prime \prime}$ near $t_{0}$ will be more $\dagger$ For the laser field $\mathcal{E}(t)=\mathcal{E}_{0} \cos (\omega t)$ and ionization at the maximum of the laser field $\mathbf{r}_{0}(t)=$ $\mathcal{E}_{0} / \omega^{2}(1-\cosh (\omega t))[13]$. 
suppressed than excitations which occur near the real time-axis, i.e. close to the exit from the barrier. The indirect ionization mechanism thus represents a pathway to the ionization of deeply bound orbitals which is not subject to the full exponential energy suppression of the direct pathway. Neglecting laser-induced dynamics in the ion for simplicity, the ratio of indirect and direct pathways leading to the same final state of the ion $m$ is given by: $e^{\Delta I_{p} \tau} \int_{0}^{\tau} d t^{\prime \prime} V_{m n}^{\text {corr }}\left(t^{\prime \prime}\right) e^{-\Delta I_{p} t^{\prime \prime}}$, where $V_{m n}^{\text {corr }}(t)=\left\langle m\left|V^{e e}\left(\mathbf{r}_{0}(t)\right)\right| n\right\rangle$ is a matrix element of electron-electron correlation operator along the subbarrier trajectory calculated between the field-free states of the ion, $\Delta I_{p}$ is the energy gap between these states, $\tau$ is the imaginary part of $t_{0}$, refereed to as tunneling time.

To gauge the importance of the indirect ionization pathway, we calculated the ratio of indirect ionization amplitude to the direct amplitude for the parent channel:

$$
\widetilde{a}_{\mathfrak{m} \mathfrak{n}}^{(i)}\left(\mathbf{k}_{f}\right)=a_{\mathfrak{m} \mathfrak{n}}^{(i)}\left(\mathbf{k}_{f}\right) / a_{\mathfrak{n}}^{(d)}\left(\mathbf{k}_{f}\right)=c_{\mathfrak{m} \mathfrak{n}}\left(\mathbf{k}_{f}\right) / b_{\mathfrak{n} \mathfrak{n}}\left(t, t_{0}\right)
$$

for $\mathrm{N}_{2}$ and $\mathrm{CO}_{2}$ molecules. Following convention [6,9], we name the essential channels $\mathrm{X}, \mathrm{A}$ and $\mathrm{B}$ in order of increasing ionization potential. Here we focus on indirect ionization pathways leading to excitation of the cation upon removing the electron from HOMO and thus contributing to ionization from deeper molecular orbitals. Deexcitation pathways, leaving the cation in the ground state upon removal of electron from deeper orbitals and thus contributing to ionization signal from HOMO will be considered elsewhere. Calculations were performed for an $800 \mathrm{~nm}$ laser with intensity $\mathrm{I}=1.710^{14}$ $\mathrm{W} / \mathrm{cm}^{2}$ for electrons leaving the molecule at the peak of the electric field.

The vertical ionization potentials for channels $\mathrm{X}, \mathrm{A}$ and $\mathrm{B}$ are known spectroscopically: $15.6 \mathrm{eV}, 16.9 \mathrm{eV}, 19.1 \mathrm{eV}$ for $\mathrm{N}_{2}$ and $13.8 \mathrm{eV}, 17.3 \mathrm{eV}, 18.1 \mathrm{eV}$ for $\mathrm{CO}_{2}$. To include the effects of the laser field, we use complete active space self-consistent field (CASSCF) values for the transition dipole matrix elements between ionic states calculated in $[6,9]$. For $\mathrm{N}_{2}$, the dipole coupling vectors between the ionic states are $d_{X A}^{C A S}=(0.25,0,0), d_{X B}^{C A S}=(0,0,0.72)$, while for $\mathrm{CO}_{2}$, they are $d_{X A}^{C A S}=(0,0,0.46)$, $d_{X B}^{C A S}=(0.27,0,0)$. The first component in the brackets is perpendicular and the last is parallel to the molecular axis. Note that the Hartree-Fock approximation often overestimates the strength of dipole couplings: for the XA transition in $\mathrm{CO}_{2}$ by a factor of 3.5, for the XB transition in $\mathrm{N}_{2}$ by a factor of 2.2. Using the Hartree Fock dipoles thus lead to a strong overestimation of laser-induced dynamics in molecular ions $\mathrm{N}_{2}$ and $\mathrm{CO}_{2}$. Because the correlation potential, calculated in the Hartree-Fock approximation as $V_{m n}^{c o r r}(t)=\int d \mathbf{r} \frac{\phi_{m}^{*}(\mathbf{r}) \phi_{n}(\mathbf{r})}{\left|\mathbf{r}_{0}(t)-\mathbf{r}\right|}, n, m=X, A, B$, asymptotes to $V_{m n}(t) \approx d_{m n} / r_{0}^{2}$ for large $r_{0}$, it is likely that this correlation potential will lead to an overestimation of correlation effects. To achieve the correct asymptotic value of the correlation potential, we scale the value of the correlation potential by the ratio of CASSCF and the Hartree-Fock transition dipoles.

Because the indirect channel results from electronic correlation, it is strongly affected by the electronic structure of the ion in the field of the laser. This may be seen in three ways. First, the enhanced exponential suppression of the excitation pathway from $t^{\prime \prime}$ to the real time-axis means that highly energetic excitations, or excitations 

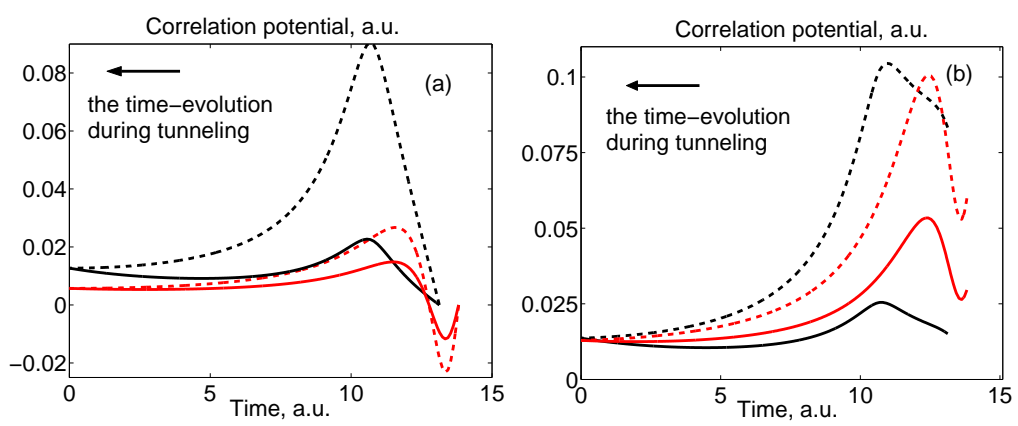

Figure 1. (a) Field-free correlation potentials $V_{A X}^{\text {corr }}(t)$ vs (imaginary) time for $\mathrm{CO}_{2}$ aligned at $0^{\circ}$ (dashed black) and $\mathrm{N}_{2}$ aligned at $90^{\circ}$ (dashed red). Solid curves show the effect of exponential suppression $V_{A X}^{\text {corr }}(t) e^{-\Delta I_{p}^{A X} t}$ vs (imaginary) time for $\mathrm{CO}_{2}$ (black) $\mathrm{N}_{2}$ ( red). Ionization starts at $\tau=13$ a.u. (b) Laser-dressed correlation potentials $V_{\mathfrak{A} \mathfrak{X}}^{\text {corr }}(t)$ with (solid) and without (dashed) exponential suppression for $\mathrm{CO}_{2}$ oriented at $0^{\circ}$ ( black) and $\mathrm{N}_{2}$ oriented at $90^{\circ}$ (red)

which occur at large imaginary times, are strongly suppressed. Second, the sign of the correlation potential may change as the electron passes through an orbital node while exiting the molecule. Third, laser-induced polarization may lead to substantial modification of orbital geometry. All of these effects may be seen by comparing $\mathrm{CO}_{2}$ to $\mathrm{N}_{2}$ at appropriate molecular orientations.

The effects of exponential suppression and laser-induced polarization may be seen by comparing $\mathrm{N}_{2}$ aligned at $90^{\circ}$ to the laser electric field to $\mathrm{CO}_{2}$ aligned at $0^{\circ}$. At both orientations, only the $\mathrm{X}$ and $\mathrm{A}$ channels contribute to ionization. Fig 1(a) shows the correlation potential calculated between field free Hartree Fock states, scaled to agree with CASSCF dipoles at long range. Dashed lines show the correlation potential, while solid lines show the correlation potential multiplied by the suppression factor $e^{-\Delta I_{p}^{A X} t}$. Due to the much smaller energy energy gap $\Delta I_{p}^{A X}$ between states $\mathrm{X}$ and $\mathrm{A}$ in $\mathrm{N}_{2}^{+}$, the correlation potential experiences much less suppression in $\mathrm{N}_{2}$ than in $\mathrm{CO}_{2}$.

The effects of laser polarization may be seen by comparing the field free correlation potential of Fig 1(a) to the one calculated using field-dressed states shown in Fig 1(b). Here the closely spaced levels of $\mathrm{N}_{2}$ result in strong orbital mixing, while the more widely spaced orbitals of $\mathrm{CO}_{2}$ are less affected. After accounting for both polarization and energy suppression, the indirect amplitude for $\mathrm{N}_{2},\left|\widetilde{a}_{\mathfrak{A} \mathfrak{X}}^{(i)}\right|=0.28$, is almost two times larger than for $\mathrm{CO}_{2},\left|\widetilde{a}_{\mathfrak{A} \mathfrak{X}}^{(i)}\right|=0.17$.

The non-scaled correlation potential (proportional to the Hartree-Fock dipole in long range) yields $\left|\widetilde{a}_{\mathfrak{A} \mathfrak{X}}^{(i)}\right|=0.5$ for $\mathrm{CO}_{2}$, reflecting exceedingly high value of $d_{X A}^{H F}$ in the Hartree-Fock approximation. A similar artefact might be responsible for strong laserinduced coupling between $\mathrm{X}$ and $\mathrm{A}$ channels in $\mathrm{CO}_{2}$ reported in Ref. [23].

In reading Figs 1,2, note that tunneling starts at (imaginary) $\tau=13$ a.u. and the (imaginary) time decreases reaching real time-axis at $t=0$, and hence the correlation potential evolves from right to left. The "later" the transition from channel X to channel 

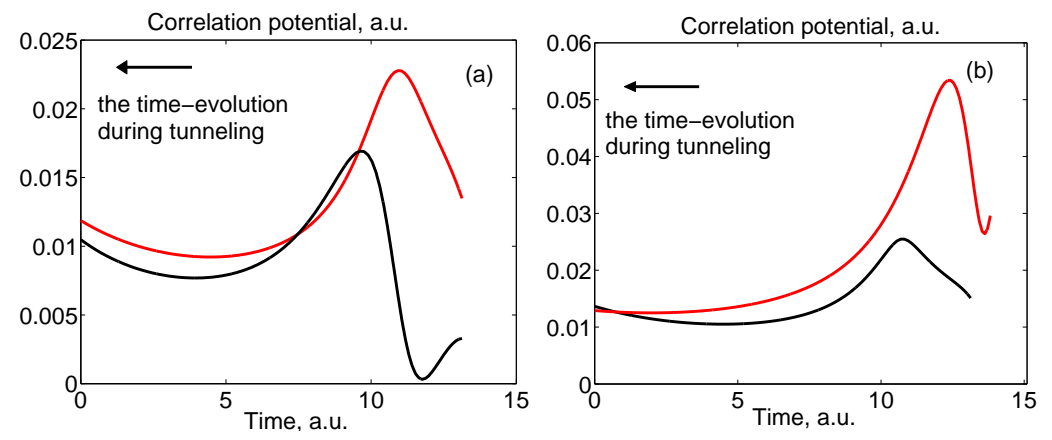

Figure 2. (a) Field-free $V_{A X}^{\text {corr }}(t)$ (red), $V_{B X}^{\text {corr }}(t)$ (black) and (b) laser dressed $V_{\mathfrak{A} \mathfrak{X}}^{\text {corr }}(t)$ (red), $V_{\mathfrak{B} \mathfrak{X}}^{\text {corr }}(t)$ (black) correlation potentials vs (imaginary) time for $\mathrm{CO}_{2}$ aligned at $30^{\circ}$.Ionization starts at $\tau=13$ a.u.

A occurs, the smaller is the effect of exponential suppression, with the correlation potential at early times $(t \sim \tau)$ suppressed the most.

The orbital nodal structure may strongly affect the temporal shape of the correlation potential. This effect may be seen by rotating $\mathrm{CO}_{2}$ molecules $30^{\circ}$ away from the laser polarization axis allowing for ionization into the $\mathrm{B}$ channel. The correlation potential $V_{B X}^{\text {corr }}$ (Figure 2(a), black curve) changes sign as the electron passes through a node in the $\mathrm{B}$ orbital. In contrast, there is no nodal plain in A orbital for the electron leaving the molecule at $30^{\circ}$ to the molecular axis and the sign of the correlation potential $V_{A X}^{\text {corr }}$ remains the same throughout (see Fig. 2(a), red curve). Because of the node in the field-free $V_{B X}^{\text {corr }}(t)$, the laser-dressed $V_{\mathfrak{B} \mathfrak{X}}^{\text {corr }}(t)$ remains small in the initial moments after ionization (Fig. 2(b), black solid curve). Since indirect ionization amplitude is controlled by the temporal structure of the laser-dressed correlation potential $V_{\mathfrak{B X}}(t)$, the effect of the node is to inhibit $\widetilde{a}_{\mathfrak{B X}}^{(i)}$ for $\mathrm{CO}_{2}$ yielding $\left|\widetilde{a}_{\mathfrak{B} \mathfrak{X}}^{(i)}\right|=0.11$.

This paper provides first theoretical analysis of attosecond dynamics of electron correlation during tunnel ionization. Calculations of indirect ionization amplitude are essentially analytic, with the ratio of indirect to direct channel given by simple integral of the correlation potential. Since multichannel strong field ionization is a key to recording ultrafast multielectron dynamics in molecules [6, 9], its understanding will have broad impact on control and imaging of these dynamics on attosecond time scale. We gratefully acknowledge stimulating discussions with M. Yu. Ivanov and SAW grant of the Leibniz society.

\section{References}

[1] Keldysh L V Sov. Physics JETP 1965201307.

[2] Rottke H, Ludwig J, and Sandner W, J. Phys. B 1996291479.

[3] Litvinyuk I V , Légaré F, Dooley P W, Villeneuve D M, Corkum P B, Zanghellini J, Pegarkov A, Fabian C, and Brabec T Phys. Rev. Lett. 20059433003 
[4] Gijsbertsen A, Boguslavskiy A, Mikosch J, Spanner M, Vrakking M, Patchkovskii S, and Stolow A submitted.

[5] Young L, Arms DA, Dufresne E M, Dunford R W, Ederer D L, Höhr C, Kanter E P, Krässig B, Landahl E C, Peterson E R, Rudati J, Santra R, and Southworth S H Phys. Rev. Lett. 2006 9783601

[6] Smirnova O, Mairesse Y, Patchkovskii S, Dudovich N, Villeneuve D, Corkum P, and Ivanov M Yu Nature 2009460972

[7] H. Akagi, Otobe T, Staudte A, Shiner A, Turner F, Dörner R, Villeneuve D M, Corkum P B Science 2009 325, 1364

[8] Haessler S, Caillat J, Boutu W, Giovanetti-Teixeira C, Ruchon T, Auguste T, Diveki Z, Breger P, Maquet A, Carré B, et al. Nature Physics, 201062002010.

[9] Mairesse Y, Higuet J, Dudovich D, Shafir D, Fabre B, Mèvel E, Constant E, Patchkovskii S, Walters Z, Ivanov M Yu, and Smirnova O Phys. Rev. Lett 2010104213601

[10] Leone S Ultrafast/Attosecond Transient Absorption with High Order Harmonics. In APS Meeting Abstracts, page 27003, 2010.

[11] Arissian L, Liu L R, Meckel M, Rippert A, Doerner R, Villeneuve D M, Staudte A Fleischer A, Woerner H J and Corkum P B submitted.

[12] Popruzhenko S V and Bauer D. Journal of Modern Optics 2008552573

[13] Perelomov A M, Popov V S, and Terent'ev M V Zhurnal Eksperimental'noi I Teoreticheskoi Fiziki, 1966501393

[14] Muth-Böhm J, Becker A, Faisal F H M Phys. Rev. Lett. 2000852280

[15] Zhao Z X, Tong X M, and Lin C D Physical Review A, 20036743404

[16] Jaron-Becker A, Becker A, Faisal F H M Phys. Rev. A, 200469023410

[17] Kjeldsen T K and Madsen L B J. Phys. B., 2004372033

[18] Smirnova O, Spanner M, Ivanov M, Journal of Modern Optics 2007541019

[19] Becker W, et al. Physical Review. A 200776033403

[20] Spanner M and Patchkovskii S Physical Review A 20098063411

[21] Gallup G A and Fabrikant I I. Physical Review A $2010 \quad 8133417$

[22] Murray R, Liu W K, Ivanov M Yu Phys. Rev. A 201081023413

[23] Petretti S, Vanne Y, Saenz A, Castro A, and Decleva P Phys. Rev. Lett. 2010104223001

[24] Becker A and Faisal F, J. Phys. B 200538 R1

[25] Ortiz J V. International Journal of Quantum Chemistry 200395593

[26] Dyson orbitals are often defined as $\left|n^{D}\right\rangle=\sqrt{N}\left\langle n^{(N-1)} \mid g^{N}\right\rangle$. We omit normalization coefficient $\sqrt{N}$ in our definition.

[27] Moiseev N, Phys. Rep. 1998302211

[28] Smirnova O, Spanner M, and Ivanov M Phys. Rev. A 20087733407

[29] Smirnova O, Mouritzen A S, Patchkovskii S, Ivanov M J. Phys. B 2007 40, F197

[30] Graefe S, Ivanov M Yu, Journal of Modern Optics 200755163603

[31] Graefe S, Ivanov M Yu, Phys. Rev. Lett 2008992527 